EPJ Web of Conferences 71, 00045 (2014)

DOI: 10.1051/epjconf/20147100045

(C) Owned by the authors, published by EDP Sciences, 2014

\title{
Simple kinetic-theory tests of dissipative and anisotropic hydrodynamics
}

\author{
Wojciech Florkowski ${ }^{1,2, a}$ \\ ${ }^{1}$ Institute of Physics, Jan Kochanowski University, PL-25406 Kielce, Poland \\ ${ }^{2}$ The H. Niewodniczański Institute of Nuclear Physics, Polish Academy of Sciences, \\ PL-31342 Kraków, Poland
}

\begin{abstract}
We solve the one-dimensional boost-invariant Boltzmann equation in the relaxation time approximation and compare the results with the predictions of dissipative and anisotropic hydrodynamics. We observe that recent formulations of second-order viscous hydrodynamics agree better with the exact solutions than the standard Israel-Stewart approach. In addition, we find that the anisotropic hydrodynamics gives a very good approximation to the exact results provided the appropriate connection between the kinetic and anisotropic hydrodynamics relaxation times is introduced.
\end{abstract}

\section{Introduction}

The production of matter in ultra relativistic heavy-ion collisions studied at RHIC and the LHC is very well described by relativistic viscous hydrodynamics [1-13]. Nevertheless, large gradients present in the early stages of the collisions suggest that viscous corrections to the ideal energy momentum tensor are quite substantial and the system's pressure is highly anisotropic.

Large pressure anisotropy leads to problems for standard 2nd-order viscous hydrodynamics [14], since it is based on a linearization around an isotropic background. Such problems triggered the development of reorganizations of viscous hydrodynamics in which large momentum-space anisotropies are built into the leading order of the hydrodynamic expansion [15-21]. The newly constructed framework is referred to as anisotropic hydrodynamics.

In this note we follow the arguments of Refs. [22, 23] and show that recent formulations of secondorder viscous hydrodynamics agree better with the exact solutions of the kinetic equation than the standard Israel-Stewart approach. We also show that the anisotropic hydrodynamics gives a very good approximation to the exact results provided the appropriate connection between the kinetic and anisotropic hydrodynamics relaxation times is introduced.

\section{Kinetic equation}

Our considerations are based on the simple form of the kinetic equation

$$
\frac{\partial f}{\partial \tau}=\frac{f^{\mathrm{eq}}-f}{\tau_{\mathrm{eq}}}
$$

\footnotetext{
ae-mail: Wojciech.Florkowski@ifj.edu.pl
} 


\section{EPJ Web of Conferences}

where $f(x, p)$ is the phase-space distribution function, $\tau=\sqrt{t^{2}-z^{2}}$ is the proper time, and $\tau_{\text {eq }}$ is the relaxation time. The requirement of boost invariance implies that $f(x, p)$ may depend only on the three variables: $\tau, w$ and $p_{T}$. The boost-invariant variable $w$ is defined by the expression

$$
w=t p_{L}-z E .
$$

Here $p_{L}\left(p_{T}\right)$ is the longitudinal (transverse) momentum and $E$ is the energy. The equilibrium background distribution function $f^{\mathrm{eq}}$ may be written in as

$$
f^{\mathrm{eq}}\left(\tau, w, p_{T}\right)=\frac{2}{(2 \pi)^{3}} \exp \left[-\frac{\sqrt{w^{2}+p_{T}^{2} \tau^{2}}}{T(\tau) \tau}\right],
$$

where $T$ is an effective temperature.

The first moment of the kinetic equation defines the divergence of the energy-momentum tensor that should be conserved.

$$
T^{\mu v}(\tau)=g_{0} \int d P p^{\mu} p^{v} f\left(\tau, w, p_{T}\right), \quad \partial_{\mu} T^{\mu v}=0 .
$$

Using the symmetry properties of the distribution function, we rewrite (4) in the form $[15,20]$

$$
T^{\mu v}=\left(\mathcal{E}+\mathcal{P}_{T}\right) u^{\mu} u^{v}-\mathcal{P}_{T} g^{\mu v}+\left(\mathcal{P}_{L}-\mathcal{P}_{T}\right) z^{\mu} z^{v}
$$

where $u^{\mu}=(t, 0,0, z) / \tau$ and $z^{\mu}=(z, 0,0, t) / \tau$. The energy density and the two (longitudinal and transverse) pressures are defined as the integrals

$$
\begin{aligned}
\mathcal{E}(\tau) & =\frac{g_{0}}{\tau^{2}} \int d P v^{2} f\left(\tau, w, p_{T}\right), \\
\mathcal{P}_{L}(\tau) & =\frac{g_{0}}{\tau^{2}} \int d P w^{2} f\left(\tau, w, p_{T}\right), \\
\mathcal{P}_{T}(\tau) & =\frac{g_{0}}{2} \int d P p_{T}^{2} f\left(\tau, w, p_{T}\right),
\end{aligned}
$$

The parameter $g_{0}$ in Eqs. (4)-(6) is the degeneracy factor connected with internal degrees of freedom different than spin, $v=\sqrt{w^{2}+p_{T}^{2} \tau^{2}}$, and $d P=d w d^{2} p_{T} / v$.

The initial condition used to solve numerically Eq. (1) corresponds to the Romatschke-Strickland form of the distribution function [24]

$$
f_{0}\left(w, p_{T}\right)=\frac{1}{4 \pi^{3}} \exp \left[-\frac{\sqrt{\left(1+\xi_{0}\right) w^{2}+p_{T}^{2} \tau_{0}^{2}}}{\Lambda_{0} \tau_{0}}\right] .
$$

This form is reduced to an isotropic Boltzmann distribution if the anisotropy parameter $\xi_{0}=\xi\left(\tau_{0}\right)$ vanishes. In this case, the transverse momentum scale $\Lambda_{0}=\Lambda\left(\tau_{0}\right)$ is equal to the system's initial temperature $T_{0}$. 


\section{Second order hydrodynamics}

In second-order viscous hydrodynamics the system's evolution is described by the energy evolution equation supplemented by the shear viscous stress evolution equation (see, for example, Ref. [2])

$$
\begin{aligned}
& \partial_{\tau} \mathcal{E}=-\frac{\mathcal{E}+\mathcal{P}}{\tau}+\frac{\Pi}{\tau}, \\
& \partial_{\tau} \Pi=-\frac{\Pi}{\tau_{\pi}}+\frac{4}{3} \frac{\eta}{\tau_{\pi} \tau}-\beta \frac{\Pi}{\tau} .
\end{aligned}
$$

Here $\mathcal{P}=\mathcal{E} / 3$ is the equilibrium pressure, $\eta$ is the viscosity coefficient, and $\tau_{\pi}=5 \bar{\eta} / T$ is the shear relaxation time ( $\bar{\eta}$ is the ratio of the viscosity to the entropy density). In most of the practical applications, the value $\beta=4 / 3$ is used which we will refer to as the Israel-Stewart (IS) prescription. We will also compare the exact solutions with the complete second-order treatment from Ref. [25] which gives $\beta=38 / 21$. We will refer to the second choice as the DNMR prescription. In both cases one can compute the transverse and longitudinal pressures using equations from the previous Section. To be consistent with the exact solution and the anisotropic hydrodynamics approximation we assume an ideal equation of state for the viscous hydrodynamical approximations. The transverse and longitudinal pressures are defined by the equations

$$
\mathcal{P}_{T}=\mathcal{P}+\frac{\Pi}{2}, \quad \mathcal{P}_{L}=\mathcal{P}-\Pi
$$

and

$$
\Pi=\frac{2}{3}\left(\mathcal{P}_{T}-\mathcal{P}_{L}\right)=\frac{4 \eta}{3 \tau} .
$$

The results obtained from (8) will be compared with the numerical solutions together with anisotropic hydrodynamics results in Sec. 5 .

\section{Anisotropic hydrodynamics}

In the anisotropic hydrodynamics approach one assumes that the distribution function is given by a spheroidal Romatschke-Strickland form (7) defined by the two time-dependent parameters: the transverse momentum scale $\Lambda(\tau)$ and the anisotropy parameter $\xi(\tau)$. The equations of anisotropic hydrodynamics for our system read [16]

$$
\begin{gathered}
\frac{\partial_{\tau} \xi}{1+\xi}=\frac{2}{\tau}-\frac{4 \mathcal{R}(\xi)}{\tau_{\mathrm{eq}}^{\mathrm{AH}}} \frac{\mathcal{R}^{3 / 4}(\xi) \sqrt{1+\xi}-1}{2 \mathcal{R}(\xi)+3(1+\xi) \mathcal{R}^{\prime}(\xi)}, \\
\frac{1}{1+\xi} \frac{\partial_{\tau} \Lambda}{\Lambda}=\frac{\mathcal{R}^{\prime}(\xi)}{\tau_{\mathrm{eq}}^{\mathrm{AH}}} \frac{\mathcal{R}^{3 / 4}(\xi) \sqrt{1+\xi}-1}{2 \mathcal{R}(\xi)+3(1+\xi) \mathcal{R}^{\prime}(\xi)},
\end{gathered}
$$

where

$$
\mathcal{R}(z)=\frac{1}{2}\left[\frac{1}{1+z}+\frac{\tan ^{-1} \sqrt{z}}{\sqrt{z}}\right]
$$

and $\tau_{\mathrm{eq}}^{\mathrm{AH}}$ is the anisotropic hydrodynamics relaxation time. We connect the relaxation time in the kinetic equation to $\tau_{\mathrm{eq}}^{\mathrm{AH}}$ by the formula $[22,23]$

$$
\tau_{\mathrm{eq}}^{\mathrm{AH}}=\frac{T}{2 \Lambda} \tau_{\text {eq }} .
$$


$\mathcal{E} / \mathcal{E}_{0}$
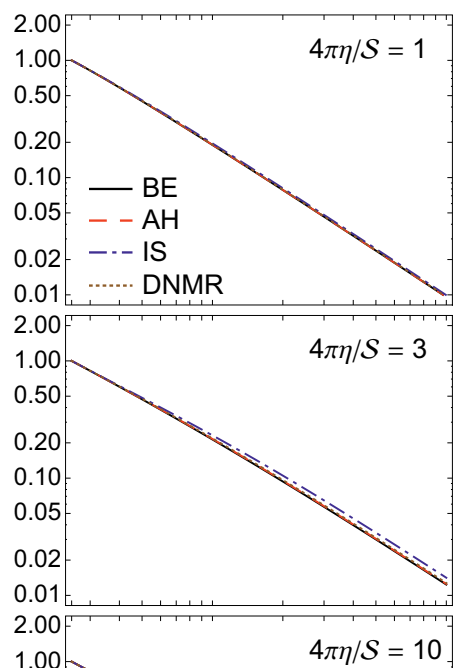

0.50

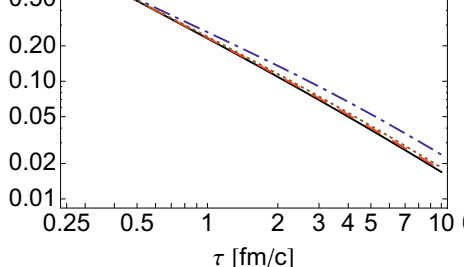

$3 \mathcal{P}_{L} / \mathcal{E}_{0}$
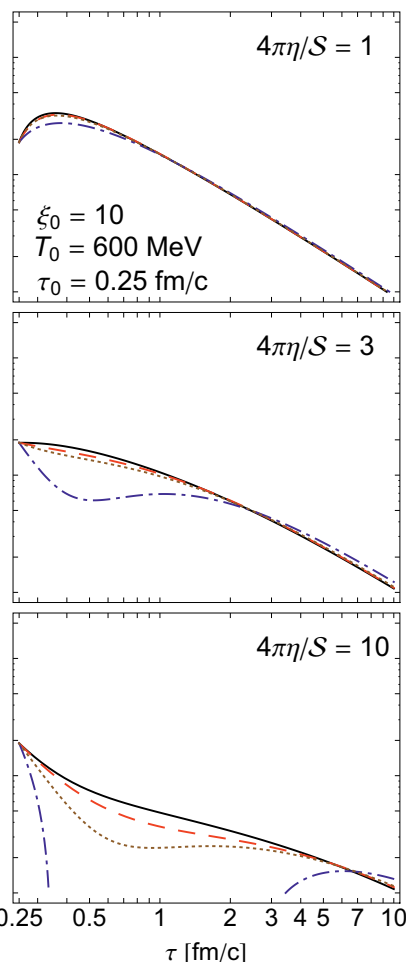

$3 \mathcal{P}_{T} / \mathcal{E}_{0}$
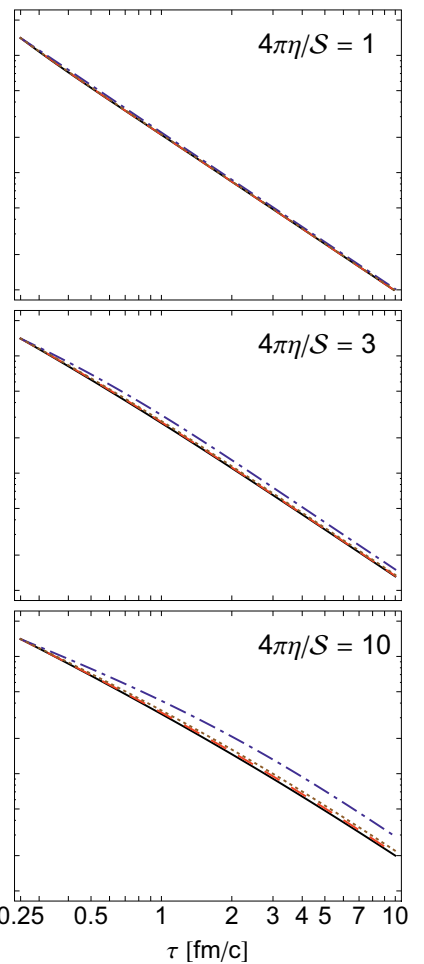

Figure 1. Time dependence of the energy density, longitudinal pressure, and transverse pressure obtained from the kinetic equation (BE), Israel-Stewart theory (IS), modern second order hydrodynamics (DNMR), and anisotropic hydrodynamics (AH). The presented results correspond to different values of the viscosity coefficient.

All physical quantities may be expressed in terms of $\Lambda(\tau)$ and $\xi(\tau)$. In particular, the two pressures are obtained from the expressions

$$
\mathcal{P}_{T}=\frac{3 g_{0} \Lambda^{4}}{\pi^{2}} \mathcal{R}_{T}(\xi), \quad \mathcal{P}_{L}=\frac{3 g_{0} \Lambda^{4}}{\pi^{2}} \mathcal{R}_{L}(\xi),
$$

where

$$
\mathcal{R}_{T}(z)=\frac{3}{2 z}\left[\frac{1+\left(z^{2}-1\right) \mathcal{R}(z)}{1+z}\right]
$$

and

$$
\mathcal{R}_{L}(z)=\frac{3}{z}\left[\frac{(1+z) \mathcal{R}(z)-1}{1+z}\right]
$$

\section{Results and Conclusions}

In Fig. 1 we present an example of our results. We show the time dependence of the energy density, the longitudinal pressure, and the transverse pressure (three columns of panels from left to right, 
respectively) obtained for three different values of the viscosity: $4 \pi \bar{\eta}=1,3,10$ (three rows of panels from top to bottom, respectively). The energy density is normalized to its initial value, while the longitudinal and transverse pressures are normalized to one third of the initial energy density. In this way, the late-time behavior of the displayed quantities becomes similar.

The initial conditions are characterized by the anisotropy parameter $\xi_{0}=10$ and the initial effective temperature $T_{0}=600 \mathrm{MeV}$. In all cases considered one observes noticeable differences between the exact results (denoted as BE) and the standard Israel-Stewart approximation (denoted as IS). If the shear viscosity becomes large, the Israel-Stewart theory results in negative longitudinal pressure.

The agreement between viscous hydrodynamics and kinetic theory is significantly improved if one uses the framework of Ref. [25] (denoted as DNMR) and is further improved if one uses the anisotropic hydrodynamics approximation (denoted as AH). We note, however, that the problem of negative pressure, although lessened somewhat, still exists in the DNMR approach.

Altogether, our results inidcate advantages of the recent formulations of viscous and anisotropic hydrodynamics as compared to the traditional Israel-Stewart approach.

Acknowledgments: This work was supported in part by the Polish National Science Center with decision No. DEC-2012/06/A/ST2/00390.

\section{References}

[1] W. Israel and J. M. Stewart, Annals Phys. 118, 341 (1979)

[2] A. Muronga, Phys. Rev. C 69, 034903 (2004)

[3] R. Baier, P. Romatschke and U. A. Wiedemann, Phys. Rev. C 73, 064903 (2006)

[4] P. Romatschke and U. Romatschke, Phys. Rev. Lett. 99, 172301 (2007).

[5] K. Dusling and D. Teaney, Phys. Rev. C 77, 034905 (2008).

[6] M. Luzum and P. Romatschke, Phys. Rev. C 78, 034915 (2008).

[7] H. Song and U. W. Heinz, J. Phys. G 36, 064033 (2009).

[8] G. S. Denicol, T. Kodama and T. Koide, J. Phys. G 37, 094040 (2010).

[9] B. Schenke, S. Jeon and C. Gale, Phys. Lett. B 702, 59 (2011).

[10] C. Shen, U. Heinz, P. Huovinen and H. Song, Phys. Rev. C 84, 044903 (2011).

[11] P. Bozek, Phys. Lett. B 699, 283 (2011).

[12] H. Niemi, G. S. Denicol, P. Huovinen, E. Molnar and D. H. Rischke, Phys. Rev. Lett. 106, 212302 (2011).

[13] P. Bozek and I. Wyskiel-Piekarska, Phys. Rev. C 85, 064915 (2012).

[14] M. Martinez and M. Strickland, Phys. Rev. C 79, 044903 (2009).

[15] W. Florkowski and R. Ryblewski, Phys. Rev. C 83, 034907 (2011).

[16] M. Martinez and M. Strickland, Nucl. Phys. A 848, 183 (2010).

[17] R. Ryblewski and W. Florkowski, J. Phys. G 38, 015104 (2011).

[18] M. Martinez and M. Strickland, Nucl. Phys. A 856, 68 (2011).

[19] R. Ryblewski and W. Florkowski, Eur. Phys. J. C 71, 1761 (2011).

[20] M. Martinez, R. Ryblewski and M. Strickland, Phys. Rev. C 85, 064913 (2012).

[21] R. Ryblewski and W. Florkowski, Phys. Rev. C 85, 064901 (2012).

[22] W. Florkowski, R. Ryblewski and M. Strickland, Nucl. Phys. A 916, 249 (2013)

[23] W. Florkowski, R. Ryblewski and M. Strickland, Phys. Rev. C 88, 024903 (2013)

[24] P. Romatschke and M. Strickland, Phys. Rev. D 68, 036004 (2003)

[25] G. S. Denicol, H. Niemi, E. Molnar and D. H. Rischke, Phys. Rev. D 85, 114047 (2012) 